\title{
e-Phaïstos
}

e-Phaïstos

Revue d'histoire des techniques / Journal of the history

of technology

IX-1 | 2021

Autour de Léonard de Vinci

\section{Léonard de Vinci : imaginaire, ingenium et conception technique}

Propos introductif

Leonardo da Vinci: Imaginary, "Ingenium" and Technical design

\section{Laure Ciccione}

\section{(2) OpenEdition}

Journals

Édition électronique

URL : https://journals.openedition.org/ephaistos/8721

DOI : 10.4000/ephaistos.8721

ISSN : 2552-0741

Éditeur

IHMC - Institut d'histoire moderne et contemporaine (UMR 8066)

Référence électronique

Laure Ciccione, «Léonard de Vinci : imaginaire, ingenium et conception technique », e-Phaïstos [En ligne], IX-1 | 2021, mis en ligne le 27 avril 2021, consulté le 17 septembre 2021. URL : http://

journals.openedition.org/ephaistos/8721 ; DOI : https://doi.org/10.4000/ephaistos.8721

Ce document a été généré automatiquement le 17 septembre 2021.

Tous droits réservés 


\title{
Léonard de Vinci : imaginaire, ingenium et conception technique
}

\author{
Propos introductif \\ Leonardo da Vinci: Imaginary, "Ingenium" and Technical design
}

\section{Laure Ciccione}

1 Léonard de Vinci n'a pas fait œuvre d'historien et ne s'est pas posé la question de l'historicité de la technique. Pourtant, le pouvoir d'attraction de son œuvre, et sa renommée si grande depuis plus de 500 ans sont nés précisément de son rapport à la technique, aux inventions et de cette recherche têtue de l'innovation. Elle remplit tout entière, ses dessins, sa peinture, ses plans, etc.

2 Alors, s'il n'est pas un historien des techniques, comment fallait-il aborder ce dossier d'e-Phaïstos qui lui est consacré?

3 La renommée du maître masquait plutôt la réalité de son œuvre : il fallait donc tâcher de s'y plonger, voir et revoir les dessins, les planches, pour s'apercevoir que cette œuvre riche et prolixe se situe au cœur d'une question fondamentale de l'histoire des techniques : celle de l'imaginaire (Garçon 2018). Car, avec Léonard, l'imaginaire joue sur deux plans, à la manière de poupées russes emboitées : il y a, d'une part, la figure mythique du maitre lui-même, façonnée par l'aura et le mystère de ses dessins comme de ses peintures. Cette image du maître, transmise de siècle en siècle jusqu'à nous, est celle d'un visionnaire un peu mystérieux, d'un précurseur de l'imaginaire technique. Pour autant, cette image constituait déjà une vision somme toute adossée à la spécificité du rapport de Léonard à la technique ; spécificité qui « fabriqua » elle-aussi « du mythe ». Reste que le dessinateur, l'artisan, le peintre, le sculpteur, etc., qu'il était, fut tout entier homme de la technique.

D'autre part, et c'est là le travail spécifique de l'historien des techniques, il y a cette interrogation sur la manière dont l'œuvre de Léonard s'est inscrit dans un imaginaire des techniques plus ancien, pour en renouveler les contours et comment cette pensée technique trouva sa place au sein d'une plus large lignée, sur la longue durée : héritages et lignée des Antiques, des machines et des objets techniques eux-mêmes, qu'ils soient 
déjà existants ou qu'ils restent eux-mêmes des objets imaginaires couchés sur le papier. Lignées aussi des savoir-faire appris dans l'atelier et de la connaissance consignée dans les bibliothèques de la Renaissance. Pouvait-on, en s'appuyant sur quelques fragments de son œuvre, comprendre plus précisément son rapport à la technique, au moment même où les Humanistes renouvelaient la lecture des Antiques?

5 S'il acquit cette connaissance livresque et humaniste qui lui fut refusée du fait de sa naissance, c'est dans l'atelier qu'il apprit les savoirs pratiques du dessin, de la peinture, la fabrication de couleurs, des liants, de la fonte du bronze, pour ne citer que ceux-là. C'est à partir de sa pratique, celle du dessin, qu'il a tenté de comprendre le monde. De la topographie d'un vaste espace géographique par exemple, d'un paysage, à la concentration sur un détail de l'anatomie humaine ou des animaux, Léonard multiplie et assemble les échelles. Tous ses dessins restituent un fragment du monde qu'il appréhende et tente de présenter dans son ensemble. Chaque détail est perçu par rapport à la compréhension de ce qui fait système. La tentative, vaine en définitive, de réunir une somme, en fait aussi un maitre de son temps.

C'est par le dessin - geste technique - et par l'observation, qu'il creusa sa propre route vers le savoir et la connaissance, qu'elle fût connaissance du monde ou de la technique proprement dite: les machines. «Léonard, le meilleur Léonard est un observateur méthodique et systématique, un "observateur-chercheur» (...). Un observateur s'appuyant sur son génie de dessinateur (...). Un visuel, doué pour la géométrie, remarquablement doué : mais la géométrie, précisément, c'est une abstraction de la vision " (Febvre 1954). C'est ainsi que Lucien Febvre concluait le colloque Léonard de Vinci et l'expérience scientifique, qu'avait organisé Alexandre Koyré en 1952. Cette pratique de Léonard (observation, sens de la vue, géométrie des machines), doit-on l'envisager comme les prémices d'un changement de paradigme autour de la profession naissante de l'ingénieur (Ravier 2017) ? Elle est, en tous les cas, porteuse d'une méthode qui n'a cessé d'être redécouverte au point de devenir une source constance d'inspiration. Pour que nombre de ses réalisations prennent vie, pour qu'elles s'animent dans le réel, il a manqué à Léonard une force, un moteur. Ce moteur, lui, il l'est devenu et l'est resté, notamment pour les ingénieurs contemporains qui voient dans ses observations une source d'inspiration au moment de concevoir les objets techniques les plus complexes.

\section{BIBLIOGRAPHIE}

Léonard de Vinci et l'expérience scientifique au XVI siècle, Colloque international, Paris, 4-7 juillet 1952, (préparé par Alexandre Koyré), Paris, Centre National de la Recherche Scientifique, 1953

FEBVRE Lucien, « Léonard de Vinci savant », Annales. Économies, sociétés, civilisations, $9^{\mathrm{e}}$ année, $\mathrm{n}^{\circ} 2$, 1954, p.238-241; DOI : 10.3406/ahess.1954.2273

GARÇON Anne-Françoise, « Du mythe au techno-imaginaire », in KRÖGER Fabian, MAESTRUTTI

Marina, Les imaginaires et les techniques, Paris, Presses des Mines, 2018, p.39-53

e-Phaïstos, IX-1 | 2021 
RAVIER-MAZZOCCO Benjamin, « La topique et ses avatars. Comment concevoir le mouvement dans une machine? ", in BOUILLON Didier, GUILLERME André, MILLE Martine et al. (dir.) Gestes techniques, techniques du geste, Villeneuve d'Ascq, Presses universitaires du Septentrion, 2017, p. 249-264 ; DOI : 10.4000/books.septentrion.12377

\section{RÉSUMÉS}

Propos introductifs à la Masterclasse "Léonard de Vinci, penseur de la technique ", organisée en 2017 par les jeunes chercheurs (masterants et doctorants) du Centre d'histoire des techniques de l'Université Paris 1 Panthéon-Sorbonne.

Introductory talk to the Masterclass "Leonardo da Vinci, thinker of technicity", organized in 2017 by the master and doctoral students of the Centre for the History of Technicity of the University of Paris 1 Panthéon-Sorbonne.

\section{INDEX}

Mots-clés : histoire des techniques, dessin, conception technique, ingénieur

Keywords : history of technology, technical drawing, technical design, engineer

\section{AUTEUR}

\section{LAURE CICCIONE}

Laure Ciccione est doctorante en histoire des techniques à l'université Paris 1 PanthéonSorbonne (ED 113/IHMC), sous la direction d'Anne-Françoise Garçon. Ses recherches portent sur les conditions d'apparition de l'automobile et les dynamiques géographiques, politiques et intersectorielles de l'innovation, à partir des archives fiscales des ateliers de métaux ou de mécanique de l'Est parisien entre 1870 et 1920. La notion d'imaginaire, interrogée à partir des sources littéraires, journalistiques et commerciales, diversifie l'approche de ces dynamiques et des reconfigurations sociales dont elles sont porteuses. 